\title{
THERMAL ANALYSIS OF CLOSED LOOP PULSATING HEAT PIPE
}

\author{
N. SANTHI SREE ${ }^{1}$, N. V. S. S. SUDHEER ${ }^{2} \&$ P. BHRAMARA ${ }^{3}$ \\ ${ }^{I}$ Institute of Aeronautical Engineering, Dundigal, Hyderabad, Telangana, India \\ ${ }^{2}$ RVRJC College of Engineering, Andhra Pradesh, India \\ ${ }^{3}$ JNTUH, Hyderabad, Telangana, India
}

\begin{abstract}
Recent advances in electronics design and manufacturing have resulted in significant increases in heat flux density through a miniaturization of components and a simultaneous increase in power requirements associated with improved product functionality. As a result, thermal management is one of the highest priority items in the production of high performance microelectronic devices. Closed loop Pulsating Heat Pipes (CLPHP) are heat exchanging devices with multiphase slug and plug flow due to capillary action. The pulsating heat pipes are miniature equipments that consist of an evaporator, condenser and adiabatic zone used for cooling purpose in electronic devices. In this present study, CLPHP's experiment is conducted by providing vital information on the parameter dependency of their thermal performance. The influence characterization has been made for variation of working fluid with filling ratios, the orientation. CLPHPs are made up of copper tubes with 2 and $3 \mathrm{~mm}$ diameters with a number of 2 tons are considered with water as the working fluid. Different heat inputs 16, 32,48W are considered for analysis. The parameters, operating between evaporator and condenser of CLPHP's are analyzed and simulation is performed using CFD. The liquid volume fractions, pressure and temperature variations among the three sections of CLPHP with different orientations of geometry have been described. Thermal resistance is compared with respect to CFD and experimental values.

KEYWORDS: Closed Loop Pulsating Heat Pipes (CLPHP), Evaporator, Condenser, CFD, Heat Flux, Heat Transfer, \& Thermal Resistance
\end{abstract}

Received: Jan 08, 2018; Accepted: Jan 29, 2018; Published: Feb 22, 2018; Paper Id.: IJMPERDAPR20183

\section{NOMENCLATURE}

D: Tube diameter

Ë̈: Eötvös number \&Bo: Bond Number

g: Gravitational acceleration

$\boldsymbol{P}:$ Electrical input power

$Q$ : Heat input

R: Resistance

$\boldsymbol{T}$ : Temperature

$T_{e}:$ Average evaporator temperature

$\boldsymbol{T}_{c}$ : Average condenser temperature 
s: Surface tension $(\mathrm{N} / \mathrm{m})$

$\boldsymbol{\rho}:$ Density $\left(\mathrm{kg} / \mathrm{m}^{3}\right)$

\section{INTRODUCTION}

In the present scenario, higher efficiency comes at a price of few glitches, which aids in describing the concept of accumulation of heat in an enclosure, potentially damaging the electrical and electronic devices. The catastrophic failure of electronics devices was caused due to overheating. Several techniques adopted for cooling include heat sinks, electric coolers, forced air systems and fans, heat pipes and others. These techniques, mostly use extended surface or fins employing conduction heat transfer mechanism. But eventually the thermal conductivity of the fin material constrains the performance. The effective thermal conductivity could be upgraded by replacing the metallic fin material by a wicked heat pipe.

A heat pipe is a heat-transfer device that combines the principles of both thermal conductivity and phase transition based on pure latent heat transfer mechanism to efficiently manage the transfer of heat between two solid interfaces. These conventional devices have been reported to be in use since last thirty years in various applications like heat exchangers, economizers, space applications and electronic cooling. But due to the miniaturization of the commodities efficiently overtaking the race, a need for betterment becomes the utmost priority which not only stands to be reliable but also within the financial limits. In addition, high heat flux removal at controlled temperature is needed for power electronics thermal management and transport requirements. These requirements pays the way for innovative heat exchanges known as Pulsating Heat Pipes (PHP) invented by Akachi et.al [1] which inculcates a strategy making use of sensible as well as latent heat transfer mechanism to accomplish effective thermal management. A PHP is essentially a non-equilibrium heat transfer device, performance success of which primarily depends on the continuous maintenance or sustenance of these non-equilibrium conditions within the system. The liquid-vaporslug transport is due to pressure pulsations in the respective tubes. There is no external power source required for fluid transport. A clear picture of the apparatus can be seen in the Figure.1.1

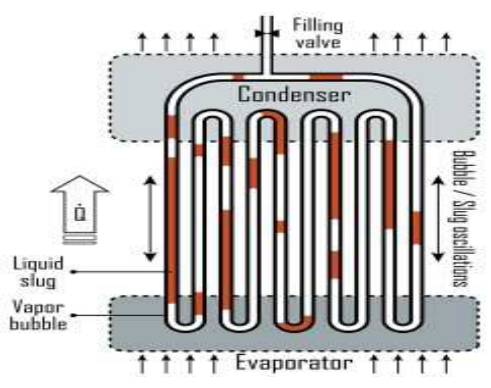

Figure 1.1: Schematic Diagram of CLPHP

\subsection{Operating Principle and Fluid Flow (Pulsating) Phenomena in PHP}

The entire essence of its thermo-mechanical physics lies in the closed (constant volume), two-phase, bubble liquid slug system, spontaneously formed inside the tube bundle, at the time of filling the device, due to the dominance of surface tension[1]. This tube bundle receives heat at one end and it is cooled at the other end. Temperature gradients give rise to temporal and spatial pressure disturbances due to resulting phase-change phenomena, i.e. generation and growth of bubbles in the evaporator and the simultaneous collapse of bubbles in the condenser [2]. The bubbles act as pumping elements, 
transporting the entrapped liquid slugs in a complex oscillating-translating-vibratory fashion, resulting in self sustained thermally driven flow oscillations and ensuing highly efficient heat transfer thereof. In addition to the latent heat, considerable amount of sensible heat transfer also occurs in a PHP. While sweeping the evaporator section, a liquid slug accumulates heat, which is eventually transferred to the condenser. The fundamental transport processes that occur inside the PHP can be understood by looking at Figure. 1.2 which suggests the various forces, including heat and mass transfer processes acting on a typical liquid-slug vapor-bubble (unit cell) system, as formed inside the PHP.

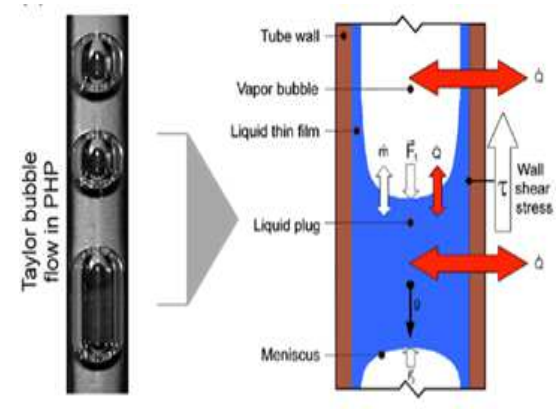

Figure 1.2: Operating Principle

\subsection{Factors Affecting Performance of PHP}

- The various factors[3] that effect the performance of PHP are

- Working Fluid

- $\quad$ Filling Ratio

- Number of Turns

- Dimensional Parameters

- Heat Flux, Orientation (Gravity effect).

\section{LITERATURE REVIEW}

Since the introduction of Pulsating Heat Pipes into the modern world by Akachi et.al where every aspect is in a verge of miniaturization, researchers have been showing a lot of interest in the development and up gradation of PHP. In this chapter some of the recent literature regarding the effect of orientation and heat flux has been discussed. Patel et.al [2] investigated the effect of heat input and orientation on performance of CLPHP by conducting experiments on a nine turn CLPHP with inner and outer diameter of copper tube $2 \mathrm{~mm}$ and $4 \mathrm{~mm}$ respectively and water as the working fluid with fill ratio 50\%. Kandekaret.al [3] provided an overview on the definition of heat pipe which summarized that three thermomechanical boundary conditions i.e., internal diameter, input heat flux and filling ratio was to be satisfied for the structure to behave as a true pulsating device. Yanget.al [4] presented an experimental study on the operation limitations of the CLPHP which consists of total 40 copper tubes with $1 \mathrm{~mm}$ and $2 \mathrm{~mm}$ inner and outer diameter respectively, with working fluid R134 and filling ratios 30\%, 50\%, 70\%. Three operational orientations were investigated viz. vertical bottom heated, horizontal heated and vertical top heated. Holleyet.al [5] studied the effect of diameter profile, gravity, and fill ratio, heating and cooling schemes on Pulsating Heat Pipe performance. Results indicate that the heat transfer can be enhanced when the diameter of the channel is varied along the channel length, thereby providing increased range of heat load 
capacity, less sensitive to gravity and in some cases smaller temperature differentials. J. L. Xuet.al [6] presented a high speed flow visualization results of the CLPHP, which identified that there exists bulk circulation flow which last longer and the local flow direction switch flow. Jioet.al [7] conducted experiment on novel cryogenic heat pipe (oscillating heat pipe) which consists of $4 \times 18.5 \mathrm{~cm}$ evaporator, $6 \times 18.5 \mathrm{~cm}$ condenser and $10 \mathrm{~cm}$ length adiabatic section and evaporator charged with liquid nitrogen at $48 \%$ fill ratio operated in horizontal direction. The experimental results depicted that the oscillating heat pipe operation changed from steady state to unsteady state until new steady state was reached. HwanKwonet.al [8] performed experiments to investigate the effect of the dual diameter channel on the flow and heat transfer characteristics of flat plate Micro Pulsating Heat Pipes (MPHP). Mameliet.al [9] experimentally investigated a multi turn CLPHP made of copper with internal and external diameter $1.1 \mathrm{~mm}$ and $2 \mathrm{~mm}$ respectively filled with FC-72. Results summarized that the CLPHP was very much sensitive to gravity head and that the vertical operation is affected by the high heat input levels.. Tonget.al [10] studied flow visualization for CLPHP using a charge coupled device. The observation reported in the paper stated that during the start-up period, working fluid oscillates with larger amplitude, however, at steady operating state the working fluid circulates. lueet.al [11] presented experimental investigation to find the effect of working fluid on thermal performance of CLPHP made of long copper capillary tubes with inner diameters of $1.0,1.5$, and $2 \mathrm{~mm}$ and bent into 32 turns. R123, ethanol, and water were selected as the working fluids with filling ratio $50 \%$. The paper summarized that the latent heat of evaporation increases in a case of the vertical CLPHP, and when the dynamic viscosity of liquid increases in a case of the horizontal CLPHP, the thermal resistance increases and the thermal performance consequently decreases since the working fluid with higher liquid viscosity requires higher driving force to overcome the friction force. lGonzalezet.al [12] experimentally investigated the performance of pulsating heat pipe (PHP) using nano fluids. The setup consisted of transparent fused quartz tubing with $3 \mathrm{~mm}$ diameter used to visualize the oscillations within the adiabatic section of the pipe.Baituleet.al [13] conducted both transient and steady state experiments on two turn CLPHP. Copper was used as the capillary tube material in the evaporator and condenser sections with inner diameter of $2 \mathrm{~mm}$ and outer diameter of $3 \mathrm{~mm}$. The total length of the CLPHP was $1080 \mathrm{~mm}$. The evaporator and condenser sections were $360 \mathrm{~mm}$ and $280 \mathrm{~mm}$ respectively. The experiments were conducted on vertical orientations for different heat loads varying from $10 \mathrm{~W}$ to $100 \mathrm{~W}$ in steps of $10 \mathrm{~W}$. The PHP was tested on Ethanol, Methanol, Acetone and Water as working fluids for different fill ratios from $0 \%$ to $100 \%$ in steps of $20 \%$. The results summarized that the filling ratio is a critical parameter, which needs to be optimized to achieve maximum thermal performance and minimum thermal resistance for a given operating condition. This experimental setup concluded that at $60 \%$ filling of PHP gave the optimum result. Zhang et.al [14] modeled a pulsating heat pipe (PHP) with open end by analyzing thin film evaporation and condensation. The heat transfer solutions were applied to the thermal model of the pulsating heat pipe and parametric study was performed. Vermaet.al [16] studied the effect of working fluid on the start-up and thermal performance in terms of thermal resistance and heat transfer coefficient of a pulsating heat pipe. Methanol and de-ionized water were selected as the working fluid. The minimum startup power for DI water was obtained at 50\% filling ratio and for methanol at $40 \%$. The optimum filling ratio in terms of minimum startup power and minimum thermal resistance was $50 \%$ for DI water and $40 \%$ for methanol.Pachgareet.al [17] conducted experimental and numerical study to investigate the thermodynamics of Closed Loop Pulsating Heat Pipe (CLPHP). The CLPHP was made of copper tubes having inner diameter of $2 \mathrm{~mm}$ and $3.6 \mathrm{~mm}$ with various working fluid viz. water, ethanol, methanol, acetone also binary mixture of water ethanol, water methanol and water acetone. The observation was the quantitative contribution of boiling point and latent heat of vaporization on the thermal performance and also the effect of surface tension and dynamic viscosity in driving the liquid slug against gravity 
in vertical position for circulation.

\section{METHODOLOGIES}

The methodology involves investigation of the effect of different orientations (vertical, horizontal and inclined at an angle $45^{\circ}$ ) of a Closed Loop Pulsating Heat Pipe (CLPHP) with particular dimension, working fluid and filling ratio on the performance. Also, it allows finding the optimum position of the CLPHP which gives minimum thermal resistance and the maximum heat transfer coefficient while determining the combined effect of heat flux and orientation on CLPHP.The results obtained by CFD analysis [16] is to be compared with experimental paper results of Pachghare et.al [17].

\section{NUMERICAL MODELING AND ANALYSIS OF CLPHP}

\subsection{Introduction}

Performance analysis of CLPHPs is done using Computational Fluid Dynamics method using commercial CFD package, ANSYS CFX version 15.0. For this, the geometry is modeled in ANSYS workbench. The experimental setup of CLPHP of the considered geometry [17] is shown in Figure 4.1. The distance between tubes is $20 \mathrm{~mm}$, diameter $2 \mathrm{~mm}$ and overall size of device is $60 \mathrm{~mm} \times 262 \mathrm{~mm}$.

The experimental setup of two turn CLPHP is shown below.

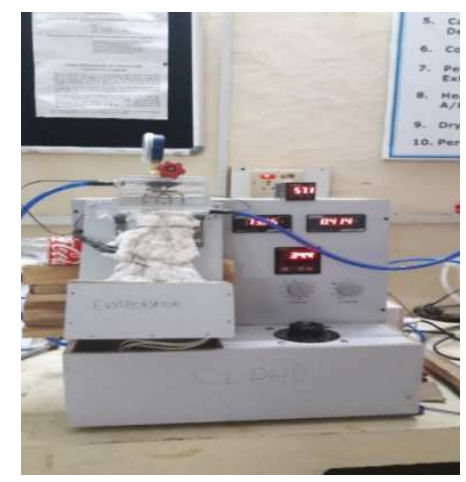

Figure 4.1: Experimental Setup of CLPHP

\subsection{Governing Equation}

The most important condition of PHP is the creation of the liquid slug. The appearance and movement of bubbles are affected by surface tension and buoyancy in the channel. Relation of surface tension and buoyancy could be explained by the dimensionless formula [10] shown in Eq. (4.1)

$$
E \delta=g \frac{D^{2}\left(\rho_{l l q}-\rho_{v a p}\right)}{\sigma}
$$

When $E \ddot{o} \approx 4$, the bubble will get seized on both sides of the wall. At this condition, the terminal velocity becomes zero and the liquid slug flow is formed. The dimensional formula for the critical diameter ' $D$ ' is given in Eq. (4.2).

$$
D<D_{\text {crit }} \leq 2 \sqrt{\frac{\sigma}{g \cdot\left(\rho_{\text {liq }}-\rho_{\text {vap }}\right)}}
$$

\subsection{Volume of Fluid Model}

The VOF formulation in ANSYS FLUENT is generally used to compute a time-dependent solution. For each 
additional phase added to the model, a variable is introduced. In each control volume, the volume fractions of all phases sum to unity. The fields for all variables and properties are shared by the phases and represent volume-averaged values, as long as the volume fraction of each of the phases is known at each location. Thus the variables and properties in any given cell are either purely representative of one of the phases, or representative of a mixture of the phases, depending upon the volume fraction values. In other words, if the $q^{\text {th }}$ fluid's volume fraction in the cell is denoted as $\alpha_{q}$, then the following three conditions are possible:

- $\boldsymbol{\alpha}_{q}=\mathbf{0}$ : The cell is empty (of the $q^{\text {th }}$ fluid).

- $\boldsymbol{\alpha}_{q}=\mathbf{1}$ : The cell is full (of the $q^{\text {th }}$ fluid).

- $\quad \mathbf{0}<\alpha_{q}<\mathbf{1}$ : The cell contains the interface between the $q^{\text {th }}$ fluid and one or more other fluids.

- Based on the local value of $\alpha_{q}$, the appropriate properties and variables will be assigned to each control volume within the domain.

\subsection{CFD Modeling and Analysis of Two Turn CLPHP}

A CLPHP has been generated with overall size of device taken as $60 \mathrm{~mm}$ x $262 \mathrm{~mm}$, distance between tubes being $20 \mathrm{~mm}$ and the diameter as $2 \mathrm{~mm}$. Since the present work focuses on the orientation of the CLPHP, the geometry is designed for all the three (Vertical, Inclined, Horizontal) cases separately with same dimensions but different planes and coordinates. CLPHP designed in the present work consists of Adiabatic, condenser and evaporator with 170, 50, $42 \mathrm{~mm}$ respectively.The geometry of the models for three different cases are shown in Figures 4.2.1-4.2.2.
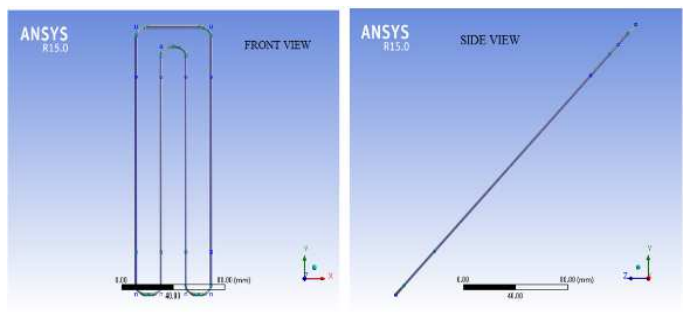

Figure 4.2.1: Geometry of the Model

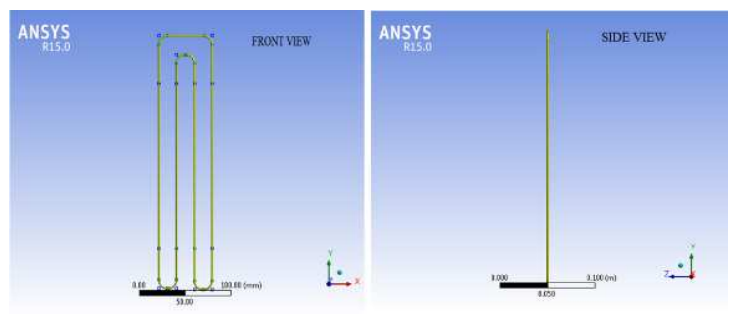

Figure 4.2.2: Geometry of the Model

\subsection{Meshing}

In this case, the automatic method for mesh shape is selected; however the sizing for the mesh is done manually. Table 4.1 depicts the number of elements and node for each case. Figure shows the meshed model for vertical, inclined and horizontal positions. The grid generation is done using cut cell method for accuracy in solution.
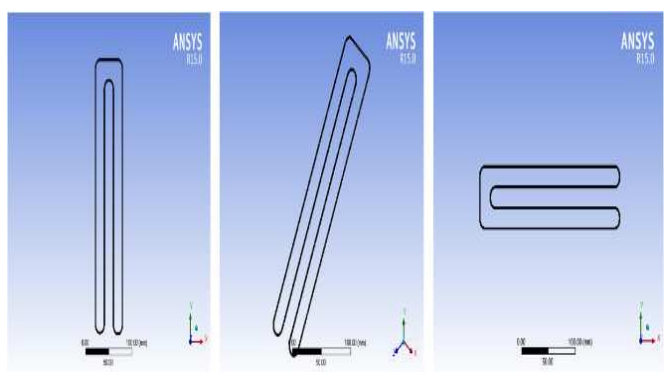

Figure 4.3: Meshing of Models (Vertical, Inclined, Horizontal) 
The discretization of a part in the model is shown in Figure. which indicates rectangular elements

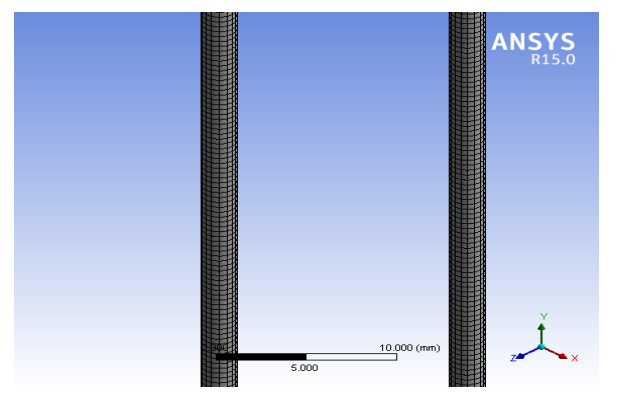

Figure 4.4: Discretization in a Part of the Geometry

The number of elements and nodes obtained due to discretization are shown below

Table 4.1: Number of Elements and Nodes

\begin{tabular}{|l|c|c|c|}
\hline \multicolumn{1}{|c|}{ Position } & Vertical & Inclined 45 $^{\circ}$ & Horizontal \\
\hline No. of nodes & 85,528 & 10,803 & 90,408 \\
\hline No. of elements & 75,674 & 15,619 & 78,180 \\
\hline
\end{tabular}

Water vapor, water liquid and air are considered as basic phases. Fluid properties are assumed to be constant with temperature. The properties of water-water vapor and air considered for the analysis. Evaporation-condensation phenomena are considered as mass transfer mechanism.

\subsection{Boundary Conditions}

- In the momentum tab, wall motion was set to stationary wall and no slip shear condition was selected for all three cases (evaporator, condenser and adiabatic).

- The adiabatic section was maintained at zero heat flux and condenser at constant temperature of $300 \mathrm{~K}$. These two boundary conditions were always the same in all the cases.

- Evaporator boundary conditions varied for different cases by changing the heat flux depending on the heat input $(16 \mathrm{~W}, 32 \mathrm{~W}, 48 \mathrm{~W})$ after deducting $10 \%$ loss of heat [17].

- The thickness of wall was mentioned as $0.0008 \mathrm{~mm}$ for every case.

\section{VALIDATION OF NUMERICAL ANALYSIS}

The simulation was run for different cases (vertical, inclined $45^{\circ}$, horizontal) at different heat inputs $(16 \mathrm{~W}, 32 \mathrm{~W}$, $48 \mathrm{~W}$ ) and thermal resistance was calculated for the same. The CFD results for the vertical operating mode is compared and validated against the results available from the experimental work [17].

\subsection{Calculation of Thermal Resistance}

The Thermal Resistance of PHP is calculated by Eq. (5.1)

$$
R_{t h}=\frac{T_{e}-T_{c}}{Q}(\mathrm{~K} / \mathrm{W}) \rightarrow(5.1)
$$

\subsection{Validation of Numerical Results}

The temperatures obtained for the evaporator and condenser section for vertical operating mode at 16W heat input 
have been listed below.

$$
\begin{aligned}
& T_{e}=331.68 \mathrm{~K} \\
& T_{c}=300 \mathrm{~K} \\
& \left.Q=P-Q_{\text {loss }} \text { (Considering the thermal losses }\right) \\
& P=16 \mathrm{~W} \\
& Q_{\text {loss }}=1.6 \mathrm{~W}(10 \% \text { of heat loss }[26]) \\
& Q=16-1.6=14.4 \mathrm{~W} \\
& R_{t h}=\frac{331.68-300}{14.4}=2.2 \mathrm{~K} / \mathrm{W} \\
& \% \text { Deviation }=\frac{R_{\text {exp }}-R}{R_{\text {exp }}} \times 100 \rightarrow(5.2)
\end{aligned}
$$

Experimental thermal resistance, $\boldsymbol{R}_{\exp }=2.6 \mathrm{~K} / \mathrm{W}$

Numerical thermal resistance, $\mathrm{R}_{\mathrm{th}}=2.2 \mathrm{~K} / \mathrm{W}$

$\%$ Deviation $=\frac{2.6-2.2}{2.6} \times 100=15.38$

The deviation calculated is $15.38 \%$ using Eq. (5.2), which is within the numerical accuracy as shown in the Figure. 5.1

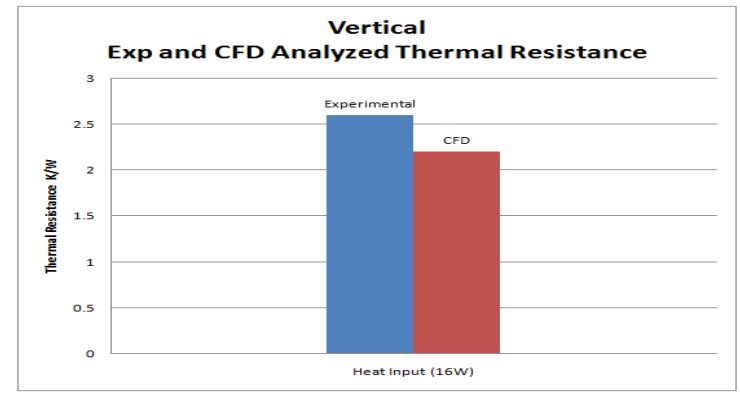

Figure 5.1: Thermal Resistance 16W Heat Input

Thermal resistance is calculated for all the heat inputs viz. $16 \mathrm{~W}, 32 \mathrm{~W}, 48 \mathrm{~W}$ using the Eq. (5.1) and weighed against the experimental results by. Pachghare et.al [17] for vertical operating mode as shown in the Figure. Thermal resistance is high at $16 \mathrm{~W}$ heat input and gradually decreases with the increase of heat flux imposed at the evaporator.

\section{RESULTS AND DISCUSSIONS}

Before getting into the results, the behavior of CLPHP can be observed from the Figure 6.1 showing the formation of vapor bubble at 2.20s in the evaporator section of inclined CLPHP (at 16W heat input) as soon as the temperature reaches $318 \mathrm{~K}$ which is the given saturation temperature. 

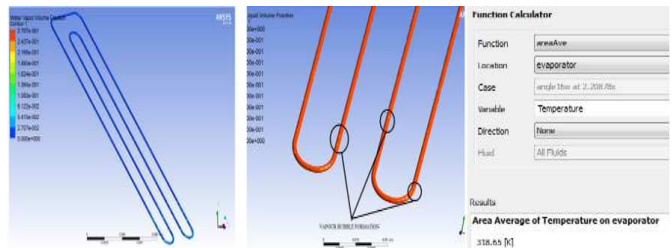

Figure 6.1: Formation of Vapour Bubble at 2.20s (Inclined Position) at 16W Heat Input

\subsection{Thermal Instability}

In order to show the occurrence of thermal instabilities during PHP operation, all the temperature signals have been plotted with time. Unstable operations occur at high heat input levels when the device is working in gravity assisted mode (Vertical bottom heating mode). However, the amplitude of oscillation increases drastically in the horizontal operating mode as shown in Figures

\subsubsection{Vertical Orientation}

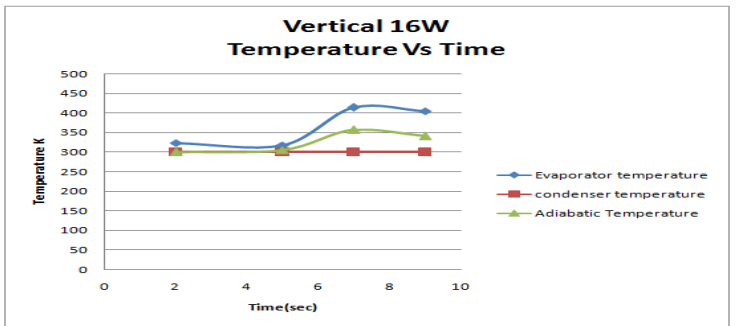

Figure 6.2: Comparison of Temperatures Over Time $(16 \mathrm{~W})$

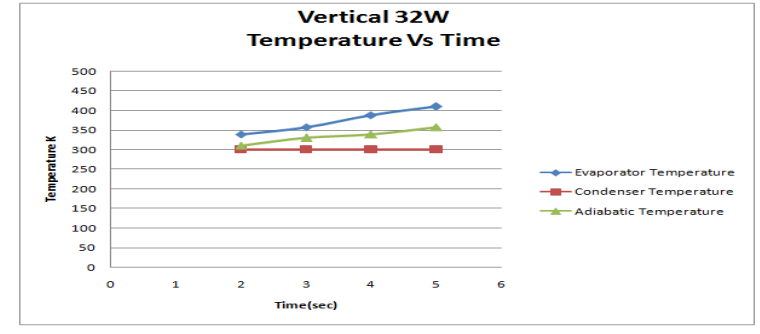

Figure 6.3: Comparison of Temperatures Over Time (32W)

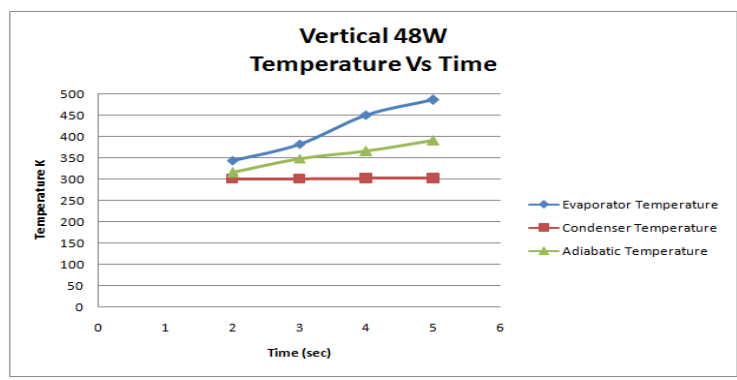

Figure 6.4: Comparison of Temperatures over Time (48W)

At heat input 16W the stability in the temperature is observed earlier as shown in Figure. when compared to the heat inputs $32 \mathrm{~W}$ and $48 \mathrm{~W}$ where the temperature is continuously shooting up as shown in the respectively. This makes it evident that thermal instability increases with the increase in heat inputs.

The horizontal operating mode was one of the most unpredictable behaviors observed in the present analysis. At $16 \mathrm{~W}$ heat input the temperature was inconsistent within the first few seconds. At $32 \mathrm{~W}$ and $48 \mathrm{~W}$ heat input also, the same scenario was observed and temperatures were comparatively high and took a long time to attain stability, compared to the other two orientations. This is due to the fact that gravity plays no role in the pulsating action in CLPHP in horizontal mode. It is also observed that the initial condition plays a dominant role in case of horizontal mode, compared to the other two orientations. 


\subsection{Water Liquid and Water Vapor Contours}

In the present analysis, three different initial conditions of the three phases inside the PHP, viz., segmental water liquid and air position, with liquid settled at the bottom of the PHP and water - liquid occupying 50\% of PHP volume, i.e. completely in the evaporator and partly in adiabatic sections. In each case, the results show rapid increase in temperature, which appeared to settle only after many time steps. Among the three initial positions considered for horizontal orientation, the segmental patching had shown better results in term slug-plug flow inside PHP. Water-liquid and water-vapour contours are obtained for vertical, inclined $45^{\circ}$ and horizontal cases at different time steps and heat input. These contours are helpful in effective visualization of the flow phenomena depicting the water and water vapour bubble movement within the tubes.

\subsubsection{Vertical Mode}

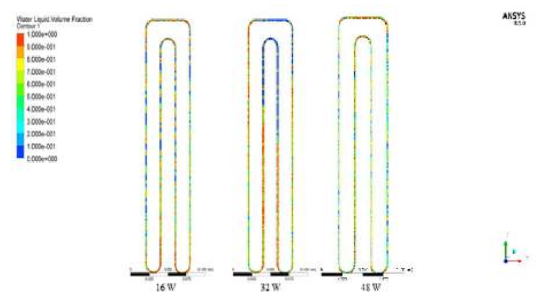

Figure 6.5: Water Liquid Volume Fraction

\subsubsection{Inclined $45^{\circ}$ Mode}

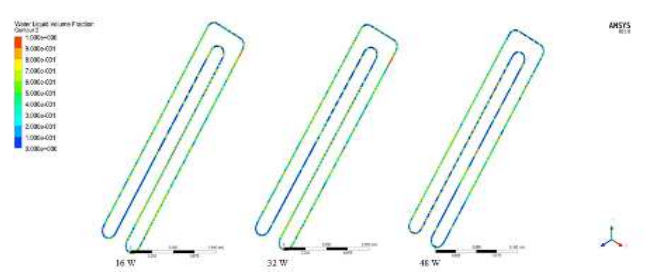

Figure 6.6: Water Liquid Volume Fraction

\subsubsection{Horizontal Mode}

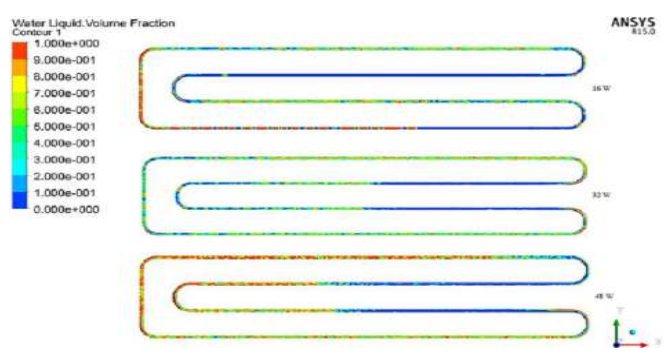

Figure 6.7: Water Liquid Volume Fraction

\subsection{Variation of Average Temperature and Pressure in each Section with Heat Input}

The following curves shows rise in average temperature, Pressure over heat input for vertical orientation 


\subsubsection{Vertical Orientation-Temperatute}

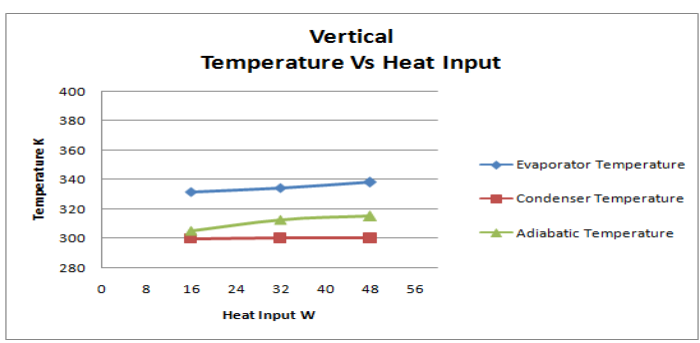

Figure: 6.8(a)

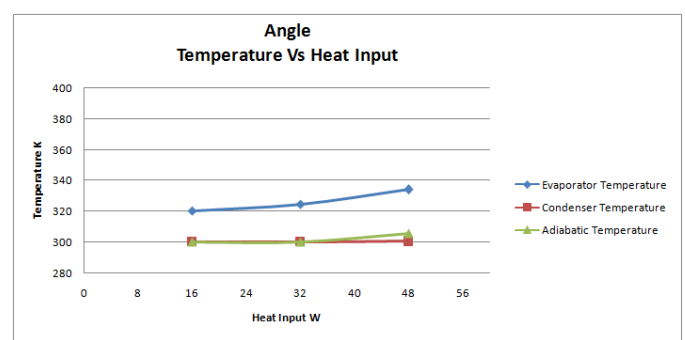

Figure: 6.8(b)

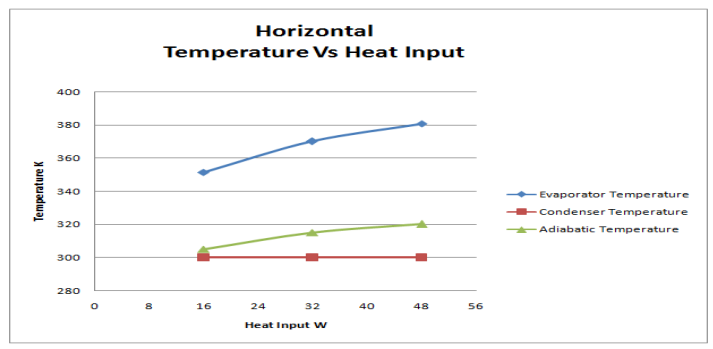

Figure: 6.8(c)

Figure 6.8: Average Temperature of each Section at different Heat Inputs ((a) Vertical, (b) Angle and(c) Horizontal Positions)

The average temperature rise in evaporator in inclined operation in less when compared to other two cases as shown in the Figure.6.8 due to the efficiency to carry the heat faster as a result of buoyancy effect.

\subsubsection{Vertical Orientation-Pressure}

\subsubsection{Condenser Section}

Pressure variation in the condenser section at four distinct planes have been plotted over time in order to study the ongoing condensation phenomena in that section at a particular time step.

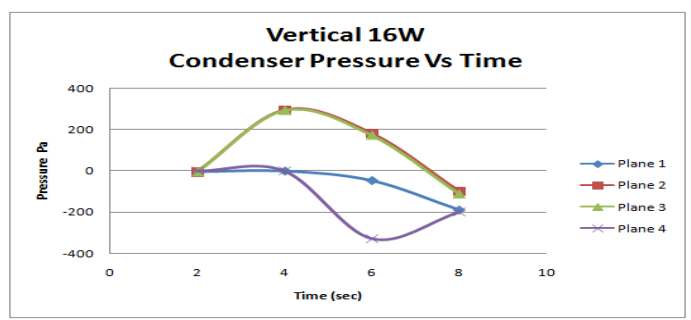

Figure 6.9: Comparison of Condenser Pressure in various Planes across Time at $16 \mathrm{~W}$

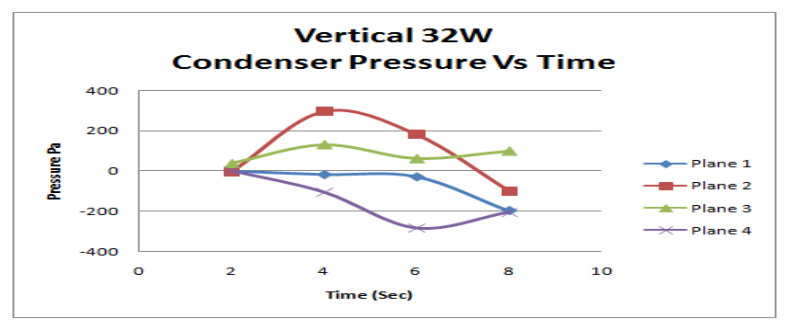

Figure 6.10 Comparison of Condenser Pressure in various Planes across Time at $32 \mathrm{~W}$

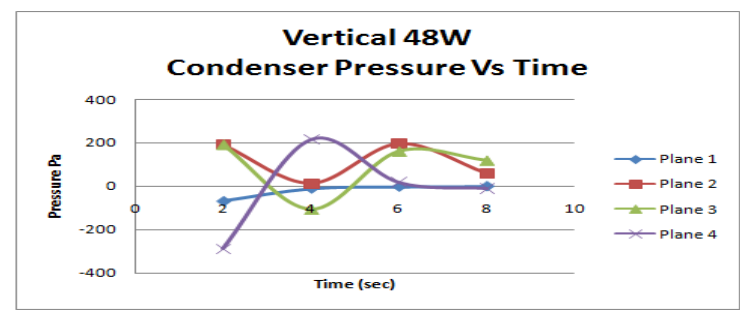

Figure 6.11: Comparison of Condenser Pressure in Various Planes across Time at $\mathbf{4 8 W}$ 
The pressure variations in the Condenser section clearly states the condensation phenomena as a dip is observed in the pressure at $6 \mathrm{sec}$ for 16W heat input in Plane 4 and Plane 1, however in Planes 2 and 3 the condensation phenomena is observed at $8 \mathrm{sec}$ as shown in the Figure. 6.9, which is due to flow fluctuations. For $32 \mathrm{~W}$ heat input the condensation has already started at $2 \mathrm{sec}$ in Plane 2 owing to the high heat input which leads to quick evaporation and high slug velocities, as shown in Figure. 6.10. Whereas the condensation phenomena starts well before 2 seconds in Planes 1 and 2, while at 4 sec in Plane 3 at 48W heat input as shown in Figure.6.11. This is due to the fact that the vapor bubble train reaches faster the condenser section compared to the other two heat inputs

\subsubsection{Adiabatic Section}

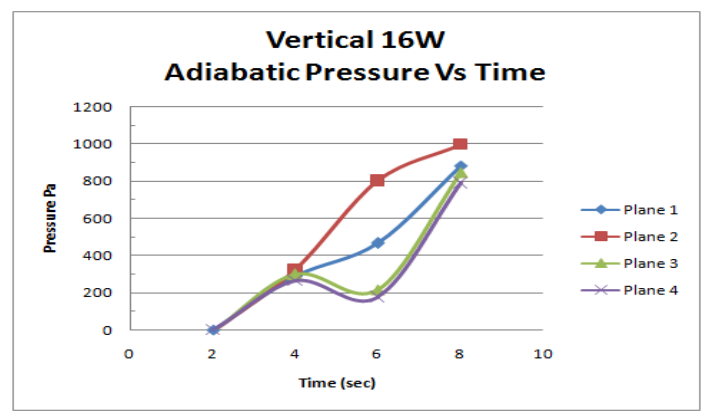

Figure 6.12: Comparison of Adiabatic Pressure in Various Planes across Time at $16 \mathrm{~W}$

Pressure variations in adiabatic section at $16 \mathrm{~W}$ indicate a uniform initial movement in the fluid starting at 2 sec through all the planes as shown in the Figure. 6.12 Since at heat distribution to the evaporator turns is uniform and at slows pace, the vapour bubble train progresses upwards causing more or less similar pressure variations in all the planes. As the time progresses, the fluctuations are seen in the adjacent legs indicating the pulsating flow. The positive pressure across all planes clearly shows the vapour formation in the evaporator section and the pumping forces, causing the upward flow.

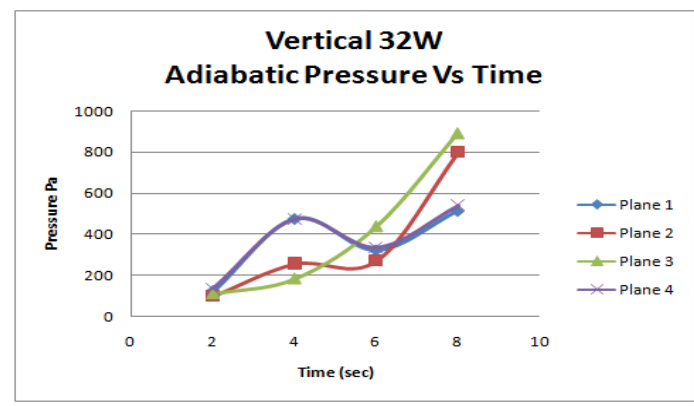

Figure 6.13: Comparison of Adiabatic Pressure in Various Planes across Time at 32W

Similar observations are found in case of heat flux of $32 \mathrm{~W}$, where the uniform pressure variation across all planes initially has turned into pulsating flow in the adjacent legs. While at $32 \mathrm{~W}$ and $48 \mathrm{~W}$ the pressure is comparatively high which indicates the vigorous movement at same time step because of high heat inputs. 


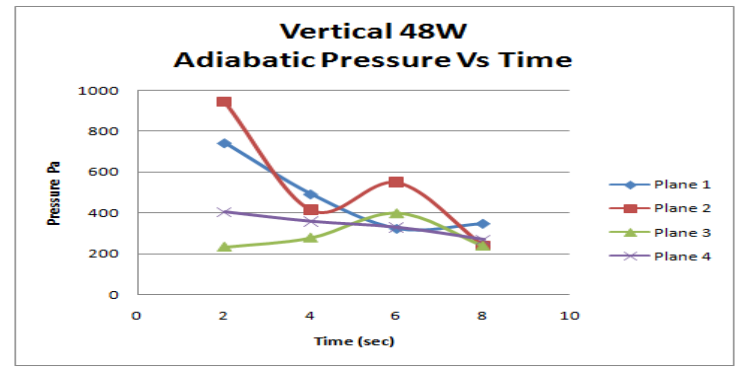

Figure 6.14: Comparison of Adiabatic Pressure in Various Planes across Time at $48 \mathrm{~W}$

\subsubsection{Evaporator Section}

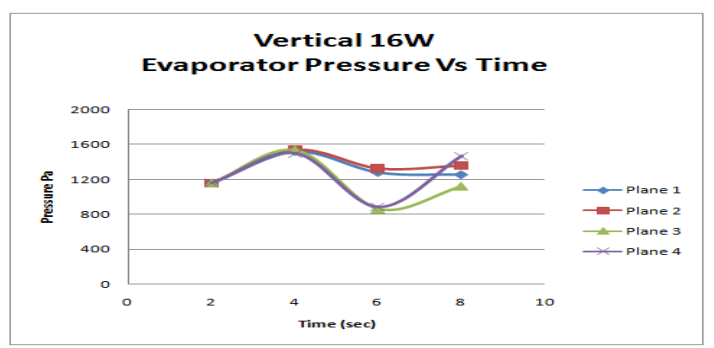

Figure 6.15: Comparison of Adiabatic Pressure in Various Planes across Time at $\mathbf{4 8 W}$

In the evaporator section at $16 \mathrm{~W}$, the pressure is observed to increase and then decrease followed by further fluctuations in the pressure as shown in Figure 6.15. These variations are the clear indication of vapour formation due to heat addition, followed by slug formation due to surface tension and capillary effects.

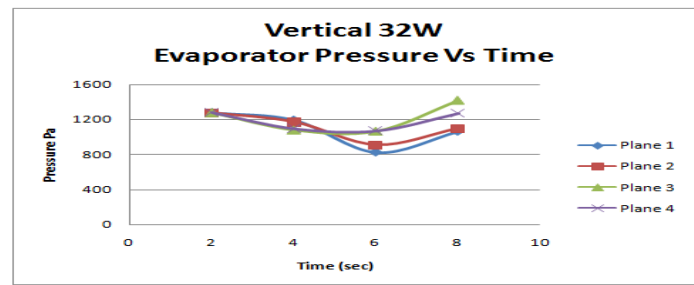

Figure 6.16: Comparison of Evaporator Pressure in Various Planes across Time at 32W

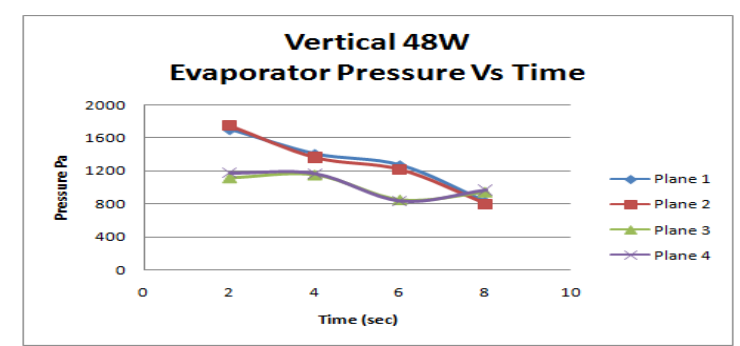

Figure 6.17: Comparison of Evaporator Pressure in Various Planes across Time at $\mathbf{4 8 W}$

Thus from the above observations of pressure variations inside the PHP at four different planes in each of condenser, adiabatic and evaporator sections, the pulsating action in PHP and the increase in pulsating behaviour with the increase of heat flux is clearly visualized. 


\subsection{Thermal Resistance of CLPHP at Different Heat Inputs}

Thermal resistance is the most important parameter for optimal PHP performance and is a function of the temperature state conditions between the evaporator and condenser. The Thermal Resistance of PHP is calculated using the Eq. (5.1).

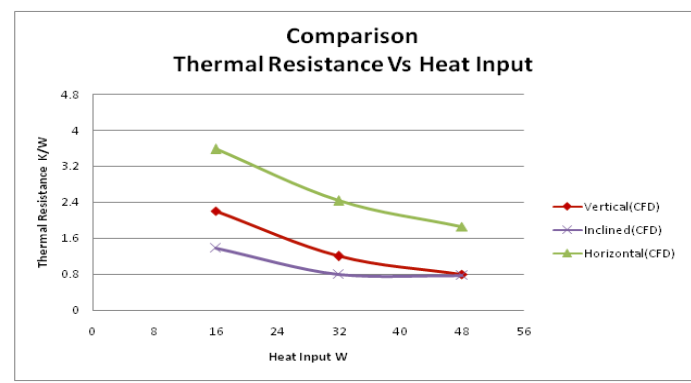

Figure 6.18: Comparison of Thermal Resistance for all the Cases at different Heat Inputs

In vertical operating mode thermal resistance is high at $16 \mathrm{~W}$ heat input and gradually decreases at $32 \mathrm{~W}$ and $48 \mathrm{~W}$. Where as in inclined operating mode, the thermal resistance is low at $16 \mathrm{~W}$ compared to vertical mode. The thermal resistance for horizontal case is very high at $16 \mathrm{~W}$ heat input when compared to the other two cases. The resistance decreases eventually at high heat inputs for all the three cases.

\section{CONCLUSIONS}

The performance characteristics have been investigated on two turn closed loops pulsating heat pipe using CFD analysis. The simulation was done for various orientations, viz, vertical, inclined at $45^{\circ}$ and horizontal, by varying the heat input. Thermal instabilities and pulsating phenomenon has been studied. Thermal Resistance is calculated for all the cases and compared against each other to find the optimum operating position. Based on the results obtained, the following conclusions are drawn.

\subsection{From the Simulation Results of PHP}

- Three phase flow in two turn closed loop pulsating heat pipe has been successfully simulated using CFD FLUENT 15.0 at different orientations, with the CFD results corroborated with that of experimental results of Pramod R. Pachghare et.al [17].

- Based on the plots of pressure and temperature variations along four different planes in the condenser, adiabatic and evaporator sections of PHP, the vapor formation due to heat addition in evaporator, the slug-plug flow, condensation in the condenser section, so caused pulsating flow and under certain situations reverse flow were clearly established.

- The pressure variations in the evaporator and condenser clearly corroborated with that of velocity variations, depicting the vaporization and condensation phenomenon respectively.

- The relative role of gravity and pressure drop on pulsating action of PHP is clearly visualized through liquid fraction and vapor fraction contours. 


\subsection{From the Comparative Results of Different Orientations}

- Thermal resistance is observed to decrease with the increase of heat flux at evaporator for all orientations.

- Among the three orientations considered in the analysis, the evaporator temperature variation is observed to increase in the horizontal operating mode; hence high thermal resistance was recorded for the same. Instabilities are observed to be higher when CLPHP operates in horizontal mode.

- Operating the CLPHP in horizontal position gave poor results since the thermal resistance obtained was high compared to other two positions.

- CLPHP gave a better performance when operated in inclined position at an angle $45^{\circ}$ to the vertical position with comparatively low thermal resistance within the operating conditions of present numerical experiments.

\section{REFERENCES}

1. Akachi, H. (1990) "Structure of Heat Pipe”, US patent, 4921041.

2. Vipul M. Patel and H. B. Mehta "Influence of Gravity on the Performance of Closed Loop Pulsating Heat Pipe" Zurich Switzerland Jan 12-13, 2016, 18 (1) Part V

3. Sameer Kandhekar and Manfred Groll "On the Definition of Pulsating Heat Pipes-An Overview" Proc. 5th Minsk International Seminar (Heat Pipes, Heat Pumps and Refrigerators), Minsk, Belarus, 2003

4. Honghai Yang, S.Khandekar, M.Groll "Operational Limit of Closed Loop Pulsating Heat Pipes" Science Direct, Applied Thermal Engineering 28 (2008) 49-59

5. Brian Holley and Amir Faghri "Analysis of pulsating heat pipe with capillary wick and varying channel diameter" International Journal of Heat and Mass Transfer 48(2005) 2635-2651

6. J. L. Xu, Y. X. Li and T. N. Wong "High Speed Visualization of Closed Loop Pulsating Heat Pipe" International Journal of Heat and Mass Transfer 48(2005) 3338-3351

7. A. L. Jiao, H.B. Ma and J. K. Crister "Experimental Investigation of Cryogenic Oscillating Heat Pipes” International Journal of Heat and Mass Transfer 52(2009) 3504-3509

8. Gi Hwan Kwon, Sung Jin Kim "Experimental Investigation on the Performance of a Micro Pulsating Heat Pipe with dual diameter" International Journal of Heat and Mass Transfer 89(2015) 817-828

9. Mauro Mameli, Vincenzo Manno, Sauro Filippeschi, Marco Marengo "Thermal instability of a Closed Loop Pulsating Heat Pipe: Combined effect of orientation and filling ratio" Experimental Thermal and Fluid Science 59(2014) 222-229

10. B. Y. Tong, T. N. Wong and K. T. Ooi “Closed-Loop Pulsating Heat Pipe” Applied Thermal Engineering 21(2001) 1845-1862

11. Niti Kammuang-lue, Phrut Sakulchangsatjatai, and Pradit Terdtoon "Effect of Working Fluids on Thermal Characteristic of a Closed-Loop Pulsating Heat Pipe Heat Exchanger: A Case of Three Heat Dissipating Devices" 978-1-4673-45521/12/\$31.00@2012 IEEE

12. G.V.R. Seshagiri Rao et al.,, Experimental Investigation of Heat Transfer Characteristics of the Heat Pipe, International Journal of Mechanical and Production Engineering Research and Development (IJMPERD), Volume 7, Issue 5, September October 2017, pp. 209-214 
13. Miguel Gonzalez and Yoon Jo Kim "Experimental Study of a Pulsating Heat Pipe Using Nanofluid as a Working Fluid" 14th IEEE ITHERM Conference,978-1-4799-5267-0/14/\$31.00 @2014 IEEE

14. Dharmapal A Baitule and Pramod R Pachghare "Experimental Analysis of Closed Loop Pulsating Heat Pipe with Variable Filling Ratio" ISSN 2278 - 0149 www.ijmerr.com Vol. 2, No. 3, July 2013 @ 2013 IJMERR

15. Yuwen Zhang and Amir Faghri "Heat Transfer in Pulsating Heat Pipe with Open End" International Journal of Heat and Mass Transfer 45(2002) 755-764

16. Jason Clement and Xia Wang "Experimental investigation of pulsating heat pipe performance with regard to fuel cell cooling application” Applied Thermal Engineering 50 (2013) 268-274

17. Umberto Costa Bitencourt, “CFD Simulation of Pulsating Heat Pipe Using ANSYS FLUENT” Technical report, Research gate, July 2016

18. Pramod R. Pachghare and Ashish M. Mahalle "Thermo-hydrodynamics of closed loop pulsating heat pipe: an experimental study” Journal of Mechanical Science and Technology 28 (8) (2014) 3387 3394, www.springerlink.com/content/1738-494x 